\title{
Extremum Seeking-based Iterative Learning Model Predictive Control (ESILC-MPC)
}

\author{
Anantharaman Subbaraman, Mouhacine Benosman
}

\begin{abstract}
In this paper, we study a tracking control problem for linear time-invariant systems, with model parametric uncertainties, under input and states constraints. We apply the idea of modular design introduced in [1], to solve this problem in the model predictive control (MPC) framework. We propose to design an MPC with input-to-state stability (ISS) guarantee, and complement it with an extremum seeking (ES) algorithm to iteratively learn the model uncertainties. The obtained MPC algorithms can be classified as iterative learning control (ILC)-MPC.
\end{abstract}

\section{INTRODUCTION}

Model predictive control (MPC), e.g., [2], is a model-based framework for optimal control of constrained multi-variable systems. MPC is based on the repeated, receding horizon solution of a finite-time optimal control problem formulated from the system dynamics, constraints on system states, inputs, outputs, and a cost function describing the control objective. However, since MPC is a model-based controller, its performance inevitably depends on the quality of the prediction model used in the optimal control computation.

In contrast, extremum seeking (ES) control is a well known approach where the extremum of a cost function associated with a given process performance (under some conditions) is found without the need for detailed modelling information, see, e.g., [3], [4], [5]. Several ES algorithms (and associated stability analysis) have been proposed, [6], [4], [7], [5], [7], [3], [8], [9], and many applications of ES have been reported [10], [11], [12], [13], [14].

The idea that we want to theoretically analyze in this paper, is that the performance of a modelbased MPC controller can be combined with the robustness of a model-free ES learning algorithm for simultaneous identification and control of linear time-invariant systems with structural uncertainties. We refer the reader to [1], [14], [15] where this idea of learning-based modular adaptive control has been introduced in a more general setting of nonlinear dynamics

A. Subbaraman (anantharaman@umail.ucsb.edu) is with the Department of Electrical Engineering, University of California at Santa Barbara, USA. M. Benosman (m_benosman@ieee.org) is with Mitsubishi Electric Research Laboratories (MERL), Cambridge, MA 02139, USA. 
We aim at proposing an alternative approach to realize an iterative learning-based adaptive MPC. We introduce an approach for an ES-based iterative learning MPC that merges a model-based linear MPC algorithm with a model-free ES algorithm to realize an iterative learning MPC that adapts to structured model uncertainties. Due to the iterative nature of the learning model improvement, we want here to compare the proposed approach to some existing Iterative learning control (ILC)-MPC methods. Indeed, ILC method introduced in [16] is a control technique which focuses on improving tracking performance of processes that repeatedly execute the same operation over time. It is of particular importance in robotics and in chemical process control of batch processes. We refer the reader to [17], [18] and [19] for more details on ILC and its applications.

At the intersection of learning based control and constrained control is the ILC-MPC concept. For instance, ILC-MPC for chemical batch processes are studied in [20], [21], and [22]. As noted in [21] one of the shortcomings of the current literature is a rigorous justification of feasibility, and Lyapunovbased stability analysis for ILC-MPC. For example, in [20] the goal is to reduce the error between the reference and the output over multiple trials while satisfying only input constraints. However, the reference signals is arbitrary and the MPC scheme for tracking such signals is not rigorously justified. Furthermore, the MPC problem does not have any stabilizing conditions (terminal cost or terminal constraint set). The ILC update law is an addition of the MPC signal of the current trial to the MPC signal of the previous trial. In [21], an ILC-MPC scheme for a general class of nonlinear systems with disturbances is proposed. The proof is presented only for MPC without constraints. In [22], the ILC update law is designed using MPC. State constraints are not considered in [22]. In [23] a batch MPC (BMPC) is proposed, which integrates conventional MPC scheme with an iterative learning scheme. A simplified static input-output map is considered in the paper as opposed to a dynamical system.

In summary, we think that there is a need for more rigorous theoretical justification attempted in this paper. Furthermore, to the best of our knowledge, the literature on ILC-MPC schemes do not consider state constraints, do not treat robust feasibility issues in the MPC tracking problem, rigorous justification of reference tracking proofs for the MPC is not present in the literature and stability proofs for the combination of the ILC and MPC schemes are not established in a systematic manner. Finally, we want to cite the work of [24], [25], [26], where similar control objectives as the one targeted in this paper, have been studied using a learning-based MPC approach. The main differences are in the control/learning design methodology and the proof techniques.

The main contribution of this work is to present a rigorous proof of an ILC-MPC scheme using existing Lyapunov function based stability analysis established in [27] and extremum seeking algorithms in 
[28], to justify the modular design method for ILC-MPC proposed in [29], where an ES-based modular approach to design ILC-MPC schemes for a class of constrained linear systems is proposed.

The rest of the paper is organized as follows. Section $\amalg$ contains some useful notations and definitions. The MPC control problem formulation is presented in Section III Section IV is dedicated to a rigorous analysis of the proposed ES-based ILC-MPC. Finally, some concluding comments are presented in Section $\mathrm{V}$

\section{NOTATION AND BASIC DEFINITIONS}

Throughout this paper, $\mathbb{R}$ denotes the set of real numbers and $\mathbb{Z}$ denotes the set of integers. State constraints and input constraints are represented by $\mathcal{X} \subset \mathbb{R}^{n}$ and $\mathcal{U} \subset \mathbb{R}^{m}$ respectively. The optimization horizon for MPC is denoted by $N \in \mathbb{Z}_{\geq 1}$. The feasible region for the MPC optimization problem is denoted by $\mathcal{X}_{N}$. A continuous function $\alpha: \mathbb{R}_{\geq 0} \rightarrow \mathbb{R}_{\geq 0}$ with $\alpha(0)=0$ belongs to class $\mathcal{K}$ if it is increasing and bounded. A function $\beta$ belongs to class $\mathcal{K}_{\infty}$ if it belongs to class $\mathcal{K}$ and is unbounded. A function $\beta(s, t) \in \mathcal{K} \mathcal{L}$ if $\beta(\cdot, t) \in \mathcal{K}$ and $\lim _{t \rightarrow \infty} \beta(s, t)=0$. Given two sets $A$ and $B$, such that $A \subset \mathbb{R}^{n}, B \subset \mathbb{R}^{n}$, the Minkowski sum is defined as $A \oplus B:=\{a+b \mid a \in A, b \in B\}$. The Pontryagin set difference is defined as $A \ominus B:=\{x \mid x \oplus B \in A\}$. Given a matrix $M \in \mathbb{R}^{m \times n}$, the set $M A \subset \mathbb{R}^{m}$, is defined as $M A \triangleq\{M a: a \in A\}$. A positive definite matrix is denoted by $P>0$. The standard Euclidean norm is represented as $|x|$ for $x \in \mathbb{R}^{n},|x|_{P}:=\sqrt{x^{T} P x}$ for a positive definite matrix $P$, $|x|_{\mathcal{A}}:=\inf _{y \in \mathcal{A}}|x-y|$ for a closed set $\mathcal{A} \subset \mathbb{R}^{n}$ and $\|A\|$ represents an appropriate matrix norm where $A$ is a matrix. $\mathbb{B}$ represents the closed unit ball in the Euclidean space. Also, a matrix $M \in \mathbb{R}^{n \times n}$ is said to be Schur iff all its eigenvalues are inside the unitary disk.

\section{PROBLEM FORMULATION}

In this section we will describe in detail the problem studied in this paper. We consider linear systems of the form

$$
\begin{aligned}
x(k+1) & =(A+\Delta A) x(k)+(B+\Delta B) u(k), \\
y(k) & =C x(k)+D u(k),
\end{aligned}
$$

where $\Delta A$ and $\Delta B$ represent the uncertainty in the system model. We will assume that the uncertainties are bounded as follows:

Assumption 1: The uncertainties $\|\Delta A\| \leq \ell_{A}$ and $\|\Delta B\| \leq \ell_{B}$ for some $\ell_{A}, \ell_{B}>0$.

Next, we impose some assumptions on the reference signal $r$.

Assumption 2: The reference signal $r:[0, T] \rightarrow \mathbb{R}$ is a piecewise constant trajectory for some $T>0$. 
Under Assumptions 1 and 2, the goal is to design a control scheme guarantying tracking with sufficiently small errors by learning the uncertain parameters of the system. Next, we will explain in detail the optimization problem associated with the MPC based controller. The results stated here are from [27]. We exploit the analysis results in [27] to establish that the closed-loop system has an ISS property with respect to the parameter estimation error.

Since the value of $\triangle A$ and $\Delta B$ are not known a priori, the MPC uses a model of the plant based on the current estimate $\hat{\Delta} A$ and $\hat{\Delta} B$.

We will now formulate the MPC problem with a given estimate of the uncertainty for a particular iteration of the learning process. We will rewrite the system dynamics as

$$
x(k+1)=f(x, u)+g(x, u, \Delta)=F(x, u, \Delta),
$$

where $f(x, u)=A x+B u$ and $g(x, u, \Delta)=\Delta A x+\Delta B u$.

Assumption 3: The state constraint set $\mathcal{X} \subset \mathbb{R}^{n}$ and control constraint set $\mathcal{U} \subset \mathbb{R}^{m}$ are compact, convex polyhedral sets.

The MPC model is generated using an estimate $\hat{\Delta} A, \hat{\Delta} B$ and is expressed as

$$
x(k+1)=f(x, u)+g(x, u, \hat{\Delta})=F(x, u, \hat{\Delta}) .
$$

We can now rewrite the actual model as

$$
x(k+1)=f(x, u)+g(x, u, \hat{\Delta})+(\Delta A-\hat{\Delta} A) x+(\Delta B-\hat{\Delta} B) u .
$$

This system can now be compared to the model in [27]. So we have

$$
x(k+1)=F(x(k), u(k), \hat{\Delta})+w(k),
$$

where

$$
w(k)=(\Delta A-\hat{\Delta} A) x(k)+(\Delta B-\hat{\Delta} B) u(k),
$$

and $x(k) \in \mathcal{X}, u(k) \in \mathcal{U}$. The following assumption will be justified in the next section.

Assumption 4: The estimates of the uncertain parameters are bounded with $\|\hat{\Delta} A\| \leq \ell_{A}$ and $\|\hat{\Delta} B\| \leq$ $\ell_{B}$ for all iterations of the extremum seeking algorithm.

We now impose certain conditions on the disturbance $w(k)$ and system matrices in accordance with [27. Assumption 1].

Assumption 5: The pair $(A+\hat{\Delta} A, B+\hat{\Delta} B)$ is controllable for every realization of $\hat{\Delta} A$ and $\hat{\Delta} B$.

We will denote the actual model using $(x, u)$ and the MPC model through $(\bar{x}, \bar{u})$. Hence we have

$$
\begin{aligned}
& x(k+1)=F(x, u, \hat{\Delta})+w, \\
& \bar{x}(k+1)=F(\bar{x}, \bar{u}, \hat{\Delta}) .
\end{aligned}
$$




\section{A. Robust positive invariant sets}

We denote the error between the states of the true model and MPC model by $e(k)=x(k)-\bar{x}(k)$. We want the error to be bounded during tracking. The error dynamics is then given by

$$
e(k+1)=(A+\hat{\Delta} A+(B+\hat{\Delta} B) K) e(k)+w(k),
$$

where $u=\bar{u}+K e$ and the matrix $K$ is such that $A_{K}:=(A+\hat{\Delta} A+(B+\hat{\Delta} B) K)$ is Schur.

We first recall the definition of a robust positive invariant set (RPI), e.g., [27].

Definition 1: A set $\Phi_{K}$ is called an RPI set for the uncertain dynamics (8), if $A_{K} \Phi_{k} \oplus \mathcal{W} \subseteq \Phi_{K}$.

So, we let $\Phi_{K}$ be an RPI set associated with the error dynamics (8), i.e., $A_{K} \Phi_{K} \oplus \mathcal{W} \subseteq \Phi_{K}$.

\section{B. Tightening the constraints}

Now we follow [27] and tighten the constraints for the MPC model so that we achieve robust constraint satisfaction for the actual model with uncertainties. Let $\mathcal{X}_{1}=X \ominus \Phi_{K}$ and $\mathcal{U}_{1}=\mathcal{U} \ominus K \Phi_{K}$. The following result is from [30, Proposition 1, Theorem 1 and Corollary 1 ].

Proposition 1: Let $\Phi_{K}$ be RPI for the error dynamics. If $e(0) \in \Phi_{K}$, then $x(k) \in \bar{x}(k) \oplus \Phi_{K}$ for all $k \geq 0$ and $w(k) \in \mathcal{W}$. If in addition, $\bar{x}(k) \in \mathcal{X}_{1}$ and $\bar{u}(k) \in \mathcal{U}_{1}$ then with the control law $u=\bar{u}+K e$, $x(k) \in \mathcal{X}$ and $u(k) \in \mathcal{U}$ for all $k \geq 0$.

\section{Invariant set for tracking}

As in [30] and [27], we will characterize the set of nominal steady states and inputs so that we can relate them later to the tracking problem. Let $z_{s}=\left(\bar{x}_{s}, \bar{u}_{s}\right)$ be the steady state for the MPC model. Then,

$$
\left[\begin{array}{cc}
A+\hat{\Delta} A-I & B+\hat{\Delta} B \\
C & D
\end{array}\right]\left[\begin{array}{l}
\bar{x}_{s} \\
\bar{u}_{s}
\end{array}\right]=\left[\begin{array}{l}
0 \\
\bar{y}_{s}
\end{array}\right]
$$

From the controllability assumption on the system matrices, the admissible steady states can be characterized by a single parameter $\bar{\theta}$ as

$$
\begin{aligned}
& \bar{z}_{s}=M_{\theta} \bar{\theta}, \\
& \bar{y}_{s}=N_{\theta} \bar{\theta},
\end{aligned}
$$

for some $\bar{\theta}$ and matrices $M_{\theta}$ and $N_{\theta}=\left[\begin{array}{ll}C & D\end{array}\right] M_{\theta}$. We let $\mathcal{X}_{s}, \mathcal{U}_{s}$ denote the set of admissible steady states that are contained in $\mathcal{X}_{1}, \mathcal{U}_{1}$ and satisfy (9). $\mathcal{Y}_{s}$ denotes the set of admissible output steady states. Now we will define an invariant set for tracking which will be utilized as a terminal constraint for the optimization problem. 
Definition 2: [27, Definition 2] An invariant set for tracking for the MPC model is the set of initial conditions, steady states and inputs (characterized by $\bar{\theta}$ ) that can be stabilized by the control law $\bar{u}=$ $\bar{K} \bar{x}+L \bar{\theta}$ with $L:=\left[\begin{array}{ll}-\bar{K} & I\end{array}\right] M_{\theta}$ while $(\bar{x}(k), \bar{u}(k)) \in \mathcal{X}_{1} \times \mathcal{U}_{1}$ for all $k \geq 0$.

We choose the matrix $\bar{K}$ such that $A_{\bar{K}}:=(A+\hat{\Delta} A+(B+\hat{\Delta} B) \bar{K})$ is Schur. We refer the reader to [30] and [27] for more details on computing the invariant set for tracking. We will refer to the invariant set for tracking as $\Omega_{\bar{K}}$. We say a point $(\bar{x}(0), \bar{\theta}) \in \Omega_{\bar{K}}$ if with the control law $u=\bar{K}\left(\bar{x}-\bar{x}_{s}\right)+\bar{u}_{s}=\bar{K} \bar{x}+L \bar{\theta}$, the solutions of the MPC model from $\bar{x}(0)$ satisfy $\bar{x}(k) \in \operatorname{Proj}_{x}\left(\Omega_{\bar{K}}\right)$ for all $k \geq 0$. As stated in [27] the set can be taken to be a polyhedral.

\section{MPC Optimization problem}

Now we will define the optimization problem that will be solved at every instant to determine the control law for the actual plant dynamics. For a given target setpoint $y_{t}$ and initial condition $x$, the optimization problem $\mathcal{P}_{N}\left(x, y_{t}\right)$ is defined as,

$$
\begin{aligned}
& \min _{\bar{x}(0), \bar{\theta}, \overline{\mathbf{u}}} V_{N}\left(x, y_{t}, \bar{x}(0), \bar{\theta}, \overline{\mathbf{u}}\right) \\
& \text { s.t } \bar{x}(0) \in x \oplus\left(-\Phi_{K}\right) \\
& \bar{x}(k+1)=(A+\hat{\Delta} A) \bar{x}(k)+(B+\hat{\Delta} B) \bar{u}(k) \\
& \bar{x}_{s}=M_{\theta} \bar{\theta} \\
& \bar{y}_{s}=N_{\theta} \bar{\theta} \\
& (\bar{x}(k), \bar{u}(k)) \in \mathcal{X}_{1} \times \mathcal{U}_{1}, k \in \mathbb{Z}_{\leq N-1} \\
& (\bar{x}(N), \bar{\theta}) \in \Omega_{\bar{K}},
\end{aligned}
$$

where the cost function is defined as follows

$$
\begin{array}{r}
V_{N}\left(x, y_{t}, \bar{x}(0), \bar{\theta}, \overline{\mathbf{u}}\right)=\sum_{k=0}^{N-1}\left|\bar{x}(k)-\bar{x}_{s}\right|_{\tilde{Q}}^{2} \\
+\left|\bar{u}(k)-\bar{u}_{s}\right|_{R}^{2}+\left|\bar{x}(N)-\bar{x}_{s}\right|_{P}^{2}+\left|\bar{y}_{s}-y_{t}\right|_{T}^{2} .
\end{array}
$$

Such cost function is frequently used in MPC literature for tracking except for the additional term in the end which penalizes the difference between the artificial stead state and the actual target value. We refer the reader to [31], [30] and [27] for more details.

Assumption 6: The following conditions are satisfied by the optimization problem

1) The matrices $\tilde{Q}>0, R>0, T>0$.

2) $(A+\hat{\Delta} A+(B+\hat{\Delta} B) K)$ is Schur matrix, $\Phi_{K}$ is a RPI set for the error dynamics, and $\mathcal{X}_{1}, \mathcal{U}_{1}$ are non-empty. 
3) The matrix $\bar{K}$ is such that $A+\hat{\Delta} A+(B+\hat{\Delta} B) \bar{K}$ is Schur and $P>0$ satisfies:

$$
\begin{array}{r}
P-(A+\hat{\Delta} A+(B+\hat{\Delta} B) \bar{K})^{T} P(A+\hat{\Delta} A+(B+\hat{\Delta} B) \bar{K})= \\
\tilde{Q}+\bar{K}^{T} R \bar{K} .
\end{array}
$$

4) The set $\Omega_{\bar{K}}$ is an invariant set for tracking subject to the tightened constraints $\mathcal{X}_{1}, \mathcal{U}_{1}$.

As noted in [27], the feasible set $\mathcal{X}_{N}$ does not vary with the set points $y_{t}$ and the optimization problem is a Quadratic programming (QP) problem. The optimal values are given by $\bar{x}_{s}^{*}, \bar{u}^{*}(0), \bar{x}^{*}$. The MPC control law writes then as: $u=\kappa_{N}(x)=K\left(x-\bar{x}^{*}\right)+\bar{u}^{*}(0)$. The MPC law $\kappa_{N}$ implicitly depends on

the current estimate of the uncertainty $\hat{\Delta}$. Also it follows from the results in [32] that the control law for the MPC problem is continuous 1 .

\section{DIRECT EXTREMUM SEEKING-BASED ITERATIVE LEARNING MPC}

\section{A. DIRECT-based iterative learning MPC}

In this section we will explain the assumptions regarding the learning cost function 2 used for identifying the true parameters of the uncertain system via nonlinear programming based extremum seeking called DIRECT, e.g., [33]. Let $\Delta$ be a vector that contains the entries in $\Delta A$ and $\Delta B$. Similarly the estimate will be denoted by $\hat{\Delta}$. Then $\Delta, \hat{\Delta} \in \mathbb{R}^{n(n+m)}$.

Since we do not impose the presence of attractors for the closed-loop system as in [34] or [35], the cost function that we utilize $Q: \mathbb{R}^{n(n+m)} \rightarrow \mathbb{R}_{\geq 0}$ depends on $x_{0}$. For iterative learning methods, the same initial condition $x_{0}$ is used to learn the uncertain parameters and hence we refer to $Q\left(x_{0}, \hat{\Delta}\right)$ as only $Q(\hat{\Delta})$ since $x_{0}$ is fixed.

Assumption 7: The learning cost function $Q: \mathbb{R}^{n(n+m)} \rightarrow \mathbb{R}_{\geq 0}$ is

1) Lipshitz in the compact set of uncertain parameters

2) The true parameter $\Delta$ is such that $Q(\Delta)<Q(\hat{\Delta})$ for all $\hat{\Delta} \neq \Delta$.

One example of a learning cost functions is identification-type cost function, where the error between outputs measurements from the system are compared to the MPC model outputs. Another example of a learning cost function, can be a performance-type cost function, where a measured output of the system is directly compared to a desired reference trajectory.

We then use the DIRECT optimization algorithm introduced in [33] for finding the global minimum of a Lipschitz function without knowledge of the Lipschitz constant. The algorithm is implemented in MATLAB using [36]. We will utilize a modified termination criterion introduced in [35] for the

\footnotetext{
${ }^{1}$ The authors would like to thank Dr. S. Di Cairano for pointing out to us the paper [32].

${ }^{2}$ Not to be confused with the MPC cost function.
} 
DIRECT algorithm to make it more suitable for extremum seeking applications. As we will mention in later sections, the DIRECT algorithm has nice convergence properties which will be used to establish our main results.

\section{B. Main results: Proof of the MPC ISS and the learning convergence}

We will now present the main results of this paper, namely the stability analysis of the proposed ILC-MPC algorithm, using the existing results for MPC tracking and DIRECT algorithm established in [27] and [28], respectively.

First, we define the value function

$$
V_{N}^{*}\left(x, y_{t}\right)=\min _{\bar{x}(0), \theta, \overline{\mathbf{u}}} V_{N}\left(x, y_{t}, \bar{x}(0), \theta, \overline{\mathbf{u}}\right)
$$

for a fixed target $y_{t}$. Also, we let $\tilde{\theta}:=\arg \min _{\bar{\theta}}\left|N_{\theta} \bar{\theta}-y_{t}\right|,\left(\tilde{x}_{s}, \tilde{u}_{s}\right)=M_{\theta} \tilde{\theta}$ and $\tilde{y}_{s}=C \tilde{x}_{s}+D \tilde{u}_{s}$. If the target steady state $y_{t}$ is not admissible, the MPC tracking scheme drives the output to converge to the point $\tilde{y}_{s}$ which is a steady state output that is admissible and also minimizes the error with the target steady state, i.e., graceful target degradation principle, e.g., [37]. The proof of the following result follows from [27, Theorem 1] and classically uses $V_{N}^{*}\left(x, y_{t}\right)$ as a Lyapunov function for the closed-loop system.

Proposition 2: Let $y_{t}$ be given. For all $x(0) \in \mathcal{X}_{N}$, the MPC problem is recursively feasible. The state $x(k)$ converges to $\tilde{x}_{s} \oplus \Phi_{K}$ and the output $y(k)$ converges to $\tilde{y}_{s} \oplus(C+D K) \Phi_{K}$.

The next result states the convergence properties of the modified DIRECT algorithm, which we will used in establishing the main result. This result is stated as [28, Assumption 7] and it follows from the analysis of the modified DIRECT algorithm in [35].

Proposition 3: For any sequence of updates $\hat{\Delta}_{t}, t=1,2, \ldots$ from the modified DIRECT algorithm and $\varepsilon>0$, there exists a $N>0$ such that $\left|\Delta-\hat{\Delta}_{t}\right| \leq \varepsilon$ for $t \geq N$.

Remark 1: Note that the results in [35] also include a robustness aspect of the DIRECT algorithm. This can be used to account for measurement noises and computational error associated with the learning cost $Q$.

We now state the main result of the section that combines the ISS MPC formulation and the extremum seeking algorithm.

Theorem 1: Under Assumptions 1-7, given an initial condition $x_{0}$, an output target $y_{t}$, such that $y_{t}$ is constant over $[0, T *]$ for some $T *$ sufficiently large. Then, for every $\varepsilon>0$, there exists $N_{1}$ and $N_{2}$ such that $\left|y(k)-\tilde{y}_{s}\right|<=\varepsilon$ for $k \in\left[N_{1}, T *\right]$ after $N_{2}$ iterations of the ILC-MPC scheme. 
Proof 1: It can observed that since the size of $\Phi_{K}$ grows with the size of $\mathcal{W}$ and $\Phi_{K}=\{0\}$ for the case without disturbances that without loss of generality $\Phi_{K} \subseteq \Gamma\left(w^{*}\right) \mathbb{B}$, where $\Gamma \in \mathcal{K}$ and $w^{*}=\| \Delta A-$ $\hat{\Delta} A\left\|X^{*}+\right\| \Delta B-\hat{\Delta} B \| U^{*}$, where $X^{*}=\max _{x \in \mathcal{X}}|x|$ and $U^{*}=\max _{u \in \mathcal{U}}|u|$. Here $X^{*}, U^{*}$ are fixed over both regular time and learning iteration number, but the uncertainties vary over iterations because of the modified DIRECT algorithm updates. Since the worst case disturbance depends directly on the estimation error, without loss of generality we have that $\Phi_{K} \subseteq \gamma(|\Delta-\hat{\Delta}|) \mathbb{B}$ and $(C+D K) \Phi_{K} \subseteq \gamma^{*}(|\Delta-\hat{\Delta}|) \mathbb{B}$ for some $\gamma, \gamma^{*} \in \mathcal{K}$. It follows from Proposition 2 that $\lim _{k \rightarrow \infty}|x(k)|_{\tilde{x}_{s} \oplus \Phi_{K}}=0$. Then,

$$
\begin{aligned}
\lim _{k \rightarrow \infty}\left|x(k)-\tilde{x}_{s}\right| & \leq \max _{x \in \Phi_{K}}|x| \\
& \leq \gamma(|\Delta-\hat{\Delta}|)
\end{aligned}
$$

We observe that the above set of equations state that the closed-loop system with the MPC controller has the asymptotic gain property and it is upper bounded by the size of the parameter estimation error. Note that the estimate $\hat{\Delta}$ is constant for a particular iteration of the process. Also, for the case of no uncertainties we have $0-$ stability (Lyapunov stability for the case of zero uncertainty). This can be proven by using the cost function $V_{N}^{*}\left(x, y_{t}\right)$ as the Lyapunov function, such that $V_{N}^{*}\left(x(k+1), y_{t}\right) \leq V_{N}^{*}\left(x(k), y_{t}\right)$ and $\lambda_{\min }(\tilde{Q})\left|x-\tilde{x}_{s}\right|^{2} \leq V_{N}^{*}\left(x, y_{t}\right) \leq \lambda_{\max }(P)\left|x-\tilde{x}_{s}\right|^{2}$, see [38]. Furthermore, here the stability and asymptotic gain property can be interpreted with respect to the compact set $\mathcal{A}:=\left\{\tilde{x}_{s}\right\}$.

Since the MPC control law is continuous, the closed-loop system for a particular iteration of the ILC-MPC scheme is also continuous with respect to the state. Then, from [39, Theorem 3.1] we can conclude that the closed-loop system is ISS with respect to the parameter estimation error and hence satisfies,

$$
\left|x(k)-\tilde{x}_{s}\right| \leq \beta\left(\left|x(0)-\tilde{x}_{s}\right|, k\right)+\hat{\gamma}(|\Delta-\hat{\Delta}|),
$$

where $\beta \in \mathcal{K} \mathcal{L}$ and $\hat{\gamma} \in \mathcal{K}$. Now, let $\varepsilon_{1}>0$ be small enough such that $\hat{\gamma}\left(\varepsilon_{1}\right) \leq \varepsilon / 2$. From Proposition 3. it follows that there exists a $N_{2}>0$ such that $\left|\Delta-\hat{\Delta}_{t}\right| \leq \varepsilon_{1}$ for $t \geq N_{2}$, where $t$ is the iteration number of the ILC-MPC scheme. Hence there exists $N_{1}>0$ such that $\left|\beta\left(\left|x(0)-\tilde{x}_{s}\right|, k\right)\right| \leq \varepsilon / 2$ for $k \geq N_{1}$. We choose $T^{*}$ such that $T^{*}>N_{1}$. Then, we have that for $k \in\left[N_{1}, T^{*}\right]$ and for $t \geq N_{2}$,

$$
\left|x(k)-\tilde{x}_{s}\right| \leq \varepsilon
$$

Similarly, using the linearity dependence between $y$ and $x$, we can also establish that, $\exists \tilde{\varepsilon}(\varepsilon)$, such that for $k \in\left[N_{1}, T^{*}\right]$ and for $t \geq N_{2}$

$$
\left|y(k)-\tilde{y}_{s}\right| \leq \tilde{\varepsilon}(\varepsilon) .
$$




\section{Conclusion}

In this paper, we have reported some results about extremum seeking-based ILC-MPC algorithms. We have argued that it is possible to merge together a model-based linear MPC algorithm with a model-free ES algorithm to iteratively learn structural model uncertainties and thus improve the overall performance of the MPC controller. We have presented the stability analysis of this modular design technique for ES-based ILC-MPC. where we addressed both feasibility and tracking performance. Future work can

include extending this method to a wider class of nonlinear systems, tracking a more richer class of signals, employing different non-smooth optimization techniques for the extremum seeking algorithm, etc.

\section{REFERENCES}

[1] M. Benosman, "Learning-based adaptive control for nonlinear systems," in European Control Conference, 2014, pp. 920-925.

[2] D. Q. Mayne, J. B. Rawlings, C. V. Rao, and P. O. M. Scokaert, "Constrained model predictive control: Stability and optimality," Automatica, vol. 36, pp. 789-814, 2000.

[3] K. B. Ariyur and M. Krstic, Real-time optimization by extremum-seeking control. John Wiley \& Sons, 2003.

[4] _ - "Multivariable extremum seeking feedback: Analysis and design," in Proc. of the Mathematical Theory of Networks and Systems, South Bend, IN, August 2002.

[5] D. Nesic, "Extremum seeking control: Convergence analysis," European Journal of Control, vol. 15, no. 34, pp. $331-347,2009$.

[6] M. Krstic, "Performance improvement and limitations in extremum seeking," Systems \& Control Letters, vol. 39, pp. 313-326, 2000.

[7] Y. Tan, D. Nesic, and I. Mareels, "On non-local stability properties of extremum seeking control," Automatica, no. 42, pp. 889-903, 2006.

[8] M. Rotea, "Analysis of multivariable extremum seeking algorithms," in Proceedings of the American Control Conference, vol. 1, no. 6. IEEE, 2000, pp. 433-437.

[9] M. Guay, S. Dhaliwal, and D. Dochain, "A time-varying extremum-seeking control approach," in American Control Conference, 2013.

[10] T. Zhang, M. Guay, and D. Dochain, "Adaptive extremum seeking control of continuous stirred-tank bioreactors," AIChE J., no. 49, p. 113123., 2003. 
[11] N. Hudon, M. Guay, M. Perrier, and D. Dochain, "Adaptive extremum-seeking control of convection-reaction distributed reactor with limited actuation," Computers \& Chemical Engineering, vol. 32, no. 12, pp. 2994 - 3001, 2008.

[12] C. Zhang and R. Ordez, Extremum-Seeking Control and Applications. Springer-Verlag, 2012.

[13] M. Benosman and G. Atinc, "Multi-parametric extremum seeking-based learning control for electromagnetic actuators," in American Control Conference, 2013.

[14] _ _ "Nonlinear learning-based adaptive control for electromagnetic actuators," in European Control Conference, 2013.

[15] G. Atinc and M. Benosman, "Nonlinear learning-based adaptive control for electromagnetic actuators with proof of stability," in IEEE, Conference on Decision and Control, 2013.

[16] S. Arimoto, "Robustness of learning control for robot manipulators," in Proceedings of the IEEE International Conference on Robotics and Automation. IEEE, 1990, pp. 1528-1533.

[17] Y. Wang, F. Gao, and F. J. Doyle III, "Survey on iterative learning control, repetitive control, and run-to-run control," Journal of Process Control, vol. 19, no. 10, pp. 1589-1600, 2009.

[18] K. L. Moore, "Iterative learning control: an expository overview," in Applied and computational control, signals, and circuits. Springer, 1999, pp. 151-214.

[19] H.-S. Ahn, Y. Chen, and K. L. Moore, "Iterative learning control: brief survey and categorization," IEEE Transactions on Systems Man and Cybernetics, vol. 37, no. 6, p. 1099, 2007.

[20] Y. Wang, D. Zhou, and F. Gao, "Iterative learning model predictive control for multi-phase batch processes," Journal of Process Control, vol. 18, no. 6, pp. 543-557, 2008.

[21] J. R. Cueli and C. Bordons, "Iterative nonlinear model predictive control. stability, robustness and applications," Control Engineering Practice, vol. 16, no. 9, pp. 1023-1034, 2008.

[22] J. Shi, F. Gao, and T.-J. Wu, "Single-cycle and multi-cycle generalized 2D model predictive iterative learning control (2D-GPILC) schemes for batch processes,' Journal of Process Control, vol. 17, no. 9, pp. 715-727, 2007.

[23] K. S. Lee, I.-S. Chin, H. J. Lee, and J. H. Lee, "Model predictive control technique combined with iterative learning for batch processes," AIChE Journal, vol. 45, no. 10, pp. 2175-2187, 1999.

[24] A. Aswani, H. Gonzalez, S. S. Sastry, and C. Tomlin, "Provably safe and robust learning-based model predictive control," Automatica, vol. 49, no. 5, pp. 1216-1226, 2013.

[25] A. Aswani, P. Bouffard, and C. Tomlin, "Extensions of learning-based model predictive control for real-time application to a quadrotor helicopter," in American Control Conference (ACC), 2012. IEEE, 2012, pp. 4661-4666. 
[26] A. Aswani, N. Master, J. Taneja, D. Culler, and C. Tomlin, "Reducing transient and steady state electricity consumption in hvac using learning-based model-predictive control," Proceedings of the IEEE, vol. 100, no. 1, pp. 240-253, 2012.

[27] D. Limon, I. Alvarado, T. Alamo, and E. Camacho, "Robust tube-based MPC for tracking of constrained linear systems with additive disturbances," Journal of Process Control, vol. 20, no. 3, pp. 248-260, 2010.

[28] S. Z. Khong, D. Nešić, Y. Tan, and C. Manzie, "Unified frameworks for sampled-data extremum seeking control: Global optimisation and multi-unit systems," Automatica, vol. 49, no. 9, pp. 27202733, 2013.

[29] M. Benosman, S. D. Cairano, and A. Weiss, "Extremum seeking-based iterative learning linear MPC," in IEEE Multi-conference on Systems and Control, 2014.

[30] I. Alvarado, D. Limon, T. Alamo, M. Fiacchini, and E. Camacho, "Robust tube based MPC for tracking of piece-wise constant references," in Decision and Control, 2007 46th IEEE Conference on. IEEE, 2007, pp. 1820-1825.

[31] I. Alvarado, D. Limon, T. Alamo, and E. Camacho, "Output feedback robust tube based MPC for tracking of piece-wise constant references," in Proceedings of the 46th IEEE Conference on Decision and Control. IEEE, 2007, pp. 2175-2180.

[32] A. Bemporad, M. Morari, V. Dua, and E. N. Pistikopoulos, "The explicit linear quadratic regulator for constrained systems," Automatica, vol. 38, no. 1, pp. 3-20, 2002.

[33] D. R. Jones, C. D. Perttunen, and B. E. Stuckman, "Lipschitzian optimization without the Lipschitz constant," Journal of Optimization Theory and Applications, vol. 79, no. 1, pp. 157-181, 1993.

[34] D. Popovic, M. Jankovic, S. Magner, and A. R. Teel, "Extremum seeking methods for optimization of variable cam timing engine operation," IEEE Transactions on Control Systems Technology, vol. 14, no. 3, pp. 398-407, 2006.

[35] S. Z. Khong, D. Nešić, C. Manzie, and Y. Tan, "Multidimensional global extremum seeking via the DIRECT optimisation algorithm,” Automatica, vol. 49, no. 7, pp. 1970-1978, 2013.

[36] D. E. Finkel, "DIRECT optimization algorithm user guide," Center for Research in Scientific Computation, North Carolina State University, vol. 2, 2003.

[37] M. Benosman and K.-Y. Lum, "On-line references reshaping and control re-allocation for nonlinear fault tolerant control," IEEE, Transactions on Control Systems Technology, vol. 17, no. 2, pp. 366379, March 2009.

[38] D. Limón, I. Alvarado, T. Alamo, and E. F. Camacho, "MPC for tracking piecewise constant 
references for constrained linear systems," Automatica, vol. 44, no. 9, pp. 2382-2387, 2008.

[39] C. Cai and A. R. Teel, "Characterizations of input-to-state stability for hybrid systems," Systems \& Control Letters, vol. 58, no. 1, pp. 47-53, 2009. 